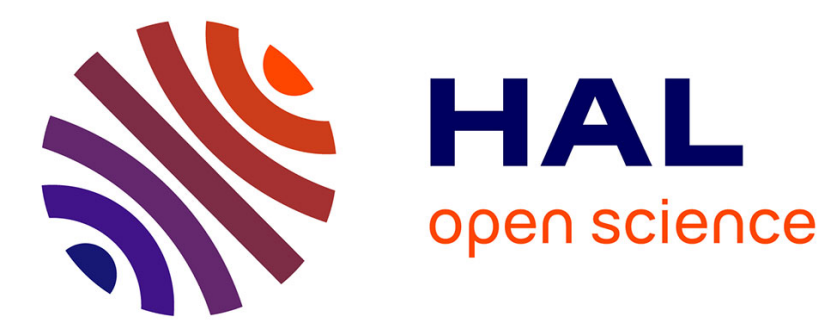

\title{
RELATIVISTIC MANY-BODY CALCULATIONS
}

W. Johnson

\section{To cite this version:}

W. Johnson. RELATIVISTIC MANY-BODY CALCULATIONS. Journal de Physique IV Proceedings, 1991, 01 (C1), pp.C1-15-C1-21. 10.1051/jp4:1991102 . jpa-00249740

\section{HAL Id: jpa-00249740 https://hal.science/jpa-00249740}

Submitted on 1 Jan 1991

HAL is a multi-disciplinary open access archive for the deposit and dissemination of scientific research documents, whether they are published or not. The documents may come from teaching and research institutions in France or abroad, or from public or private research centers.
L'archive ouverte pluridisciplinaire HAL, est destinée au dépôt et à la diffusion de documents scientifiques de niveau recherche, publiés ou non, émanant des établissements d'enseignement et de recherche français ou étrangers, des laboratoires publics ou privés. 


\section{RELATIVISTIC MANY-BODY CALCULATIONS}

\section{W.R. JOHNSON}

Département de Recherche Fondamentale, Service de Physique Atomique, Centre d'Etudes Nucléaires de Grenoble, 85X, F-38041 Grenoble, France Department of Physics, University of Notre Dame, Notre Dame, IN 46556 , U.S.A.

Résumé - On presenté des calculs à N-corps relativistes appliqués aux énergies, probabilités de transition et constantes hyperfines. Le cas des atomes et ions avec un electron de valence est plus particulièrement discuté.

Abstract - Recent relativistic many-body calculations of energies, transition amplitudes and hyperfine constants are reviewed, with emphasis on the properties of atoms and ions having a single valence electron.

\section{1 - INTRODUCTION}

This lecture gives a brief overview of work on relativistic many-body theory carried out over the past few years at Notre Dame University by Jonathan Sapirstein, Steven Blundell, Zu-Wei Liu and myself. Recently we have been joined in some of this work by Claude Guet and Jean-Paul Desclaux at Grenoble. Our concern is the structure of atoms and ions of high nuclear charge. We have in mind doing precise calculations of amplitudes of paritynonconserving transitions in atoms, such as cesium and thallium, in support of experimental efforts to determine weak interaction coupling constants, on the one hand; and calculating precise energies of highly charged ions, such as lithium-like uranium, in support of measurements of radiative corrections in strong fields, on the other hand. These problems deal with systems of high nuclear charge and are, therefore, most appropriately treated relativistically:

High $Z$ Atoms and Ions $\rightarrow$ Dirac Equation.

Moreover, our desire to treat the structure of these multi-electron systems precisely requires careful consideration of the e-e interaction:

$$
\text { More than One Electron } \rightarrow \text { Many-Body Theory. }
$$

Before elaborating on the many-body aspect of our work, let me say a few words about the use of the Dirac equation in the independent-particle approximation.

\section{2 - INDEPENDENT-PARTICLE APPROXIMATION}

If we let $\alpha$ and $\beta$ designate $4 \times 4$ Dirac matrices, let $v_{\text {nuc }}(r)$ be the nuclear potential, and introduce the central potential, $U(r)$, to account for the e-e interaction approximately, then we may write the single-particle Dirac hamiltonian governing an independent-particle (IP) state as:

$$
\mathrm{h}_{\mathrm{O}}=\mathrm{c} \alpha \cdot \mathrm{p}+\beta \mathrm{mc} \mathrm{c}^{2}+\mathrm{v}_{\mathrm{nuc}}(\mathrm{r})+\mathrm{U}(\mathrm{r}) .
$$


The orbital wave function for an electron in the state "k" is determined by solving the single-particle Dirac equation,

$$
\mathrm{h}_{0} \psi_{k}(r)=\epsilon_{k} \psi_{k}(r) \text {, }
$$

From many points of view, the best IP potential is the Hartree-Fock potential. While this potential is extremely useful in determining the structure of atoms and ions qualitatively, it is only of marginal value in precise quantitative studies. This point is illustrated in Table I, where we present energies and hyperfine constants determined for several states of lithium and cesium using (Dirac) Hartree-Fock wave functions.

Table I. Independent-Particle (Hartree-Fock) calculations of removal energies(a.u.) and hyperfine constants(MHz) for states of lithium and cesium.

\begin{tabular}{lcccrr}
\hline Element & State & $\epsilon_{H F}$ & Experiment & ${ }^{a_{H F}}$ & $a_{\exp }$ \\
\hline Lithium & $2 \mathrm{~s}_{1 / 2}$ & -0.19632 & -0.198142 & 284.34 & 401.75 \\
& $2 \mathrm{p}_{1 / 2}$ & -0.12864 & -0.130236 & 32.29 & 46.17 \\
& $2 \mathrm{p}_{3 / 2}$ & -0.12864 & -0.130235 & 6.43 & -3.07 \\
\multirow{2}{*}{ Cesium } & $6 \mathrm{~s}_{1 / 2}$ & -0.12737 & -0.14310 & 1435 & 2298 \\
& $7 \mathrm{~s}_{1 / 2}$ & -0.05519 & -0.05865 & 394 & 546 \\
\hline \hline
\end{tabular}

The Hartree-Fock energies for the $n=2$ states of 1 ithium are found to agree with the spectroscopic measurements to about $1 \%$, but the calculated hyperfine constants are in error by $25 \%$. For the $n=6$ and 7 states of cesium. the situation is seen to be even worse; the energies are in error by 10-15\%, and the hyperfine constants differ from experiment by $20-40 \%$. It is clear from examples such as these, that the independent-particle approximation is inadequate for spectroscopic purposes and that many-body effects must be taken into account.

\section{3 - APPROXIMATE MANY-BODY HAMILTONIAN FROM QED}

In order to carry out conventional many-body calculations, we need a manybody hamiltonian. The hamiltonian that we use as a point of departure for our many-body calculations is the no-pair hamiltonian from QED discussed in recent years by Sucher(1) and Mittleman(2):

$$
\begin{aligned}
H & =H_{0}+V, \\
H_{0} & =\sum_{i} \epsilon_{i} a_{i}^{\dagger} a_{i}, \\
V & =\frac{1}{2} \sum_{i j k \ell}\left[g_{i j k \ell}+b_{i j k \ell}\right] a_{i}^{\dagger} a_{j}^{\dagger} a_{k} a_{\ell}-\sum_{i j} U_{i j} a_{i}^{\dagger} a_{j},
\end{aligned}
$$

where $g_{i j k l}$ and $b_{i j k l}$ are unsymmetrized two-particle matrix elements of the Coulomb and Breit interactions, respectively, 


$$
\begin{aligned}
& \mathrm{g}_{i j k \ell}=\left\langle i j\left|\frac{\mathrm{e}^{2}}{\mathrm{r}_{12}}\right| \mathrm{k} \ell\right\rangle \\
& \mathrm{b}_{\mathrm{ijk} \ell}=\left\langle i \mathrm{j}\left|-\frac{\mathrm{e}^{2}}{2 \mathrm{r}_{12}}\left(\alpha_{1} \cdot \alpha_{2}+\alpha_{1} \cdot \hat{\mathrm{r}}_{12} \alpha_{2} \cdot \hat{\mathrm{r}}_{12}\right)\right| \mathrm{k} \ell\right\rangle
\end{aligned}
$$

The creation and annihilation operators, $a_{i}^{\dagger}$ and and $a_{j}$ in the no-pair hamiltonian refer to electron states only. The single-particle hamiltonian $\mathrm{H}_{\mathrm{O}}$ contains the eigenvalues of the one-electron Dirac equation with potential $U(r)$ written down previously; a counter-term constructed from $U(r)$ is subtracted from the interaction hamiltonian, $V$. Effects of virtual pairs are ignored in $H$, as are the effects of the retardation of the electromagnetic interaction. Such QED effects must be considered separately.

\section{4 - PERTURBATION THEORY (with $U=\mathrm{V}_{\mathrm{HF}}$ )}

The perturbation expansion of many-body theory takes its simplest form when the central potential defining the basic one-electron orbitals is the Hartree-Fock potential. For this case, the contributions from the lowest few orders of perturbation theory to the state vector of an atom or ion with one valence electron outside closed shells is given in second quantization as:

$$
\begin{aligned}
& \Psi_{0}=a_{v}^{\dagger}\left|o_{c}\right\rangle, \\
& \Psi_{1}=\sum_{m n a} \frac{g_{m n a v}}{\epsilon_{m}^{+} \epsilon_{n}-\epsilon_{a}^{-} \epsilon_{v}} a_{a} a_{v} a_{n}^{\dagger} a_{m}^{\dagger} \Psi_{0} \\
& +\frac{1}{2} \sum_{\text {mnab }} \frac{g_{m n a b}}{\epsilon_{m}^{+} \epsilon_{n}-\epsilon_{a}^{-} \epsilon_{b}} a_{a} a_{b} a_{n}^{\dagger} a_{m}^{\dagger} \Psi_{0}, \\
& \Psi_{2}=\cdots .
\end{aligned}
$$

The vector designated by $\left|{ }^{0} \mathrm{C}\right\rangle$ is the atomic core state obtained by operating on the vaccum state with a product of creation operators; one for each core electron. The first-order correction to the state vector contains only terms associated with two particle excitations; either the valence electron, $v$, and one core electron, $a$, are excited to states $n$ and $m$; or $t w o$ core electrons, $a$ and $b$, are excited to states $n$ and $m$. The second-order wave function contains 32 terms, it is written out in detail in Ref.(3) and will not be repeated here.

The corresponding perturbation expansion for the ionization energy of the valence electron is:

$$
\begin{aligned}
& E_{v}^{(0)}=\epsilon_{v}, \\
& E_{v}^{(1)}=0 . \\
& E_{v}^{(2)}=-\sum_{a m n} \frac{g_{v a m n}\left(g_{m n v a}-g_{m n a v}\right)}{\epsilon_{m}+\epsilon_{n}-\epsilon_{v}-\epsilon_{a}}+\sum_{a b m} \frac{g_{a b m v}\left(g_{m v a b}-g_{m v b a}\right)}{\epsilon_{m}+\epsilon_{v}-\epsilon_{a}-\epsilon_{b}} .
\end{aligned}
$$


It is a difficult numerical task to evaluate the first term in the expression for the second-order energy since it is neccessary to carry out double sums over single-electron states, including both bound and continuum states for all possible angular momenta. To aid in the calculation, we create a pseudospectrum for the Dirac equation by expanding the one-electron orbitals in a finite basis constructed from B-splines(4). The second term is simpler to treat since it involves only a single sum over excited states. To illustrate the extent to which many-body calculations account for the problems illustrated in Table $I$, we show in Table II the results of second-order calculations for states of 1 ithium(5). The 1\% errors in Table I are reduced to $0.1 \%$ in Table II, after second-order corrections are included.

Table II. Second-order corrections to the Dirac-Fock energies of several states in lithium determined using B-splines.

\begin{tabular}{|c|c|c|c|c|}
\hline Term & $2 s_{1 / 2}$ & $3 \mathbf{s}_{1 / 2}$ & $2 p_{1 / 2}$ & $2 p_{3 / 2}$ \\
\hline$E_{\text {doub }}^{(2)}$ & -0.002190 & -0.000473 & -0.001440 & -0.001440 \\
\hline$E_{\text {sing }}^{(2)}$ & 0.000541 & 0.000123 & 0.000065 & 0.000065 \\
\hline$E^{(2)}$ & -0.001649 & -0.000350 & -0.001374 & -0.001375 \\
\hline$E^{(0)}$ & -0.196320 & -0.073801 & -0.128638 & -0.128636 \\
\hline$E^{(0)}+E^{(2)}$ & -0.197970 & -0.074151 & -0.130012 & -0.130011 \\
\hline Exp. & -0.198142 & -0.074182 & -0.130236 & -0.130235 \\
\hline
\end{tabular}

Another illustration of the effect of second-order corrections is given in Table III, where the ionization energies of low-lying states of cesium are compared with measurement. Again, it is found that approximately $90 \%$ of the correlation energy (the difference between Hartree-Fock calculations and the actual energy) is accounted for by second-order perturbation theory.

Table III. Ionization energies for states of cesium calculated with second-order perturbation theory.

\begin{tabular}{lcccc}
\hline \hline State & $E^{(0)}$ & $E^{(2)}$ & $E^{(0)}+E^{(2)}$ & $\operatorname{Exp}$. \\
\hline $6 s_{1 / 2}$ & -0.12737 & -0.01775 & -0.14512 & -0.14310 \\
$6 p_{1 / 2}$ & -0.08562 & -0.00691 & -0.09253 & -0.09217 \\
$6 p_{3 / 2}$ & -0.08378 & -0.00618 & -0.08997 & -0.08964 \\
$5 d_{3 / 2}$ & -0.06442 & -0.01116 & -0.07558 & -0.07704 \\
$5 d_{5 / 2}$ & -0.06453 & -0.01084 & -0.07537 & -0.07659 \\
$7 s_{1 / 2}$ & -0.05519 & -0.00420 & -0.05939 & -0.05865 \\
\hline \hline
\end{tabular}

Theoretical predictions of atomic properties other than the ionization energy are also dramatically improved when higher-order corrections are taken into account. To illustrate this point, we consider the hyperfine constants of lithium and cesium, which were poorly described in the Hartree-Fock 
approximation. In Table IV, we give the second-and third-order corrections to the hyperfine constants of the states considered in Table I. (In assigning an order to a particular term in the perturbation expansion of a matrix element, we adopt the standard convention of assigning order 1 to the matrix element evaluated with $0-t h$ order wave functions.) The second-order corrections in Table IV are, in fact, iterated to include all terms of higher-order associated with the RPA (random-phase approximation). The third-order corrections include the dominant Brueckner-orbital corrections, but not the smaller third-order corrections associated with structural radiation and normalization. We see, on comparing Tables I and IV, that the $20-40 \%$ errors found in the Hartree-Fock calculations are reduced to the level of $5 \%$ after correlation corrections are considered.

Table IV. Hyperfine constants( $\mathrm{MHz})$ for states of I i thium and cesium.

\begin{tabular}{llrrrrr}
\hline \hline Element & State & \multicolumn{1}{c}{$\mathrm{a}^{(1)}$} & \multicolumn{1}{c}{$\mathrm{a}^{(2)}$} & \multicolumn{1}{c}{$\mathrm{a}^{(3)}$} & Sum & Exp. \\
\hline Lithium & $2 \mathrm{~s}_{1 / 2}$ & 284.34 & 105.53 & 10.00 & 399.87 & 401.75 \\
& $2 \mathrm{p}_{1 / 2}$ & 32.29 & 11.61 & 2.01 & 45.91 & 46.17 \\
& $2 \mathrm{p}_{3 / 2}$ & 6.46 & -10.57 & 0.40 & -3.71 & -3.07 \\
& & & & & & \\
Cesium & $6 \mathrm{~s}_{1 / 2}$ & 1435 & 289 & 642 & 2366 & 2298 \\
& $7 \mathrm{~s}_{1 / 2}$ & 394 & 80 & 98 & 572 & 546 \\
\hline \hline
\end{tabular}

In Table $V$, reduced matrix elements for the resonance transitions in cesium are tabulated and again it is found that large errors in the Hartree-Fock approximation are reduced to the level of a few percent after correlations are considered. From the results for cesium presented in Tables IV and V, it is seen that the third-order Brueckner-orbital corrections are larger than the second-order RPA corrections; calling into question the convergence of the perturbation expansion for this element. While the agreement with experiment of properties calculated using perturbation theory is encouraging. it is clearly necessary to do calculations of even higher order to determine whether or not the perturbation expansion is actually converging.

Table V. Reduced matrix elements for the resonance transitions in cesium atoms calculated in third order.

\begin{tabular}{lrcrrr}
\hline \hline Transition & ${ }_{\mathrm{D}}(1)$ & ${ }_{\mathrm{d}}{ }^{(2)}$ & $\mathrm{D}^{(3)}$ & \multicolumn{1}{c}{ Sum } & Exp. \\
\hline $6 \mathrm{~s} \rightarrow 6 \mathrm{p}_{1 / 2}$ & 5.278 & -0.303 & -0.582 & 4.393 & $4.52(1)$ \\
$6 \mathrm{~s} \rightarrow 6 \mathrm{p}_{3 / 2}$ & -7.426 & 0.413 & 0.842 & -6.171 & $-6.36(1)$ \\
\hline
\end{tabular}

\section{5 - HIGHER-ORDER PERTURBATION THEORY}

To investigate the convergence of the perturbation expansion, we undertook calculations of the ionization energy in third and fourth order. The thirdorder perturbation calculations are considerably more difficult than the corresponding second-order calculations, because of the larger number of terms to be considered and because of the added complexity of each individual term. Instead of the four terms in the second-order energy, there are fifty-six terms in the third-order energy. The most difficult term to 
evaluate is

$$
\sum_{\text {anmrs }} \frac{\left(g_{\text {avsr }}-g_{\text {vasr }}\right) g_{n m v a} g_{r s n m}}{\left(\epsilon_{n^{+}} \epsilon_{m^{-}} \epsilon_{a^{-} \epsilon_{v}}\right)\left(\epsilon_{r}{ }^{+} \epsilon_{s}-\epsilon_{a^{-}} \epsilon_{v}\right)}
$$

This term involves sums over four excited states $n, m, r, s$. If only 6 angular momenta are considered and 40 basis functions used for each angular momentum, then the sum will contain $10^{8}$ terms. Treatment of such a large scale summation requires numerical skill and large scale computing resources. Our third-order calculations were all carried out on the NCSA CRAY/XMP computer. An example of a third-order calculation for lithium is given in Table VI. The energies are seen to be in even better accord with experiment than the second-order calculations; of course the effects of the Breit interaction must be considered in order to correctly predict the fine-structure interval.

Table VI. Third-order energies for $n=2$ states of lithium.

\begin{tabular}{lccccc}
\hline \hline State & $\mathrm{E}^{(0)}$ & $\mathrm{E}^{(2)}$ & $\mathrm{E}^{(3)}$ & Sum & Exp. \\
\hline $2 \mathrm{~s}_{1 / 2}$ & -0.196320 & -0.001649 & -0.000125 & -0.198094 & -0.198142 \\
$2 \mathbf{p}_{1 / 2}$ & -0.128638 & -0.001375 & -0.000145 & -0.130158 & -0.130236 \\
$2 \mathbf{p}_{3 / 2}$ & -0.128636 & -0.001374 & -0.000145 & -0.130155 & -0.130235 \\
\hline
\end{tabular}

Calculations such as these have been carried out along the entire lithium isoelectronic sequence(6); they are, for higher $Z$ members of the sequence, of sufficiently high quality to isolate the effects of radiative corrections. To obtain such precise values for cesium(7), it is necessary to take account of the more important fourth-order terms. We obtain, for example, for the ionization energy of the $6 s_{1 / 2}$ state of cesium:

$$
\begin{aligned}
E^{(1)}+\cdots+E^{(4)} & =-0.14291 \text {, whereas, } \\
& =-0.14310 .
\end{aligned}
$$

In view of the computational difficulty and the slowness of convergence of order-by-order perturbation theory it is clearly desirable to explore methods for isolating terms containing the important physical corrections and treating such terms exactly (to all-orders in perturbation theory).

\section{6 - ALL-ORDERS CALCULATIONS}

Recently we have undertaken a series of all-orders calculations of atomic properties using an atomic state vector that is expanded as a sum of single and double excitations of the unperturbed atomic state $(8,9)$ :

$$
\begin{aligned}
\Psi=[1 & +\sum_{m a} \rho_{m a} a_{m}^{\dagger} a_{a}+\frac{1}{2} \sum_{m n a b} \rho_{m n a b} a_{m}^{\dagger} a_{n}^{\dagger} a_{b} a_{a} \\
& \left.+\sum_{m \neq v} \rho_{m v} a_{m}^{\dagger} a_{v}+\sum_{m n a} \rho_{m n v a} a_{m}^{\dagger} a_{n}^{\dagger} a_{a} a_{v}\right] \Psi_{0} .
\end{aligned}
$$


This state vector is substituted into the Schrodinger equation to obtain a set of coupled algebreic equations for the expansion coefficients. These coupled equations are solved iteratively, leading to results valid to allorders in perturbation theory. Corrections from triple excitations, for example, are not included in this wave function, and must be picked up in perturbation theory. The results of an all-orders calculation for lithium( 9 ) are given in Table VII, where the energy and hyperfine constants of the levels studied in Table I are reconsidered.

Table VII. Ali-orders calculations of removal energies(a.u.) and hyperfine constants(MHz) for states of lithium.

\begin{tabular}{lccrr}
\hline \hline State & $\mathrm{E}^{\mathrm{th}}$ & $\mathrm{E}^{\exp }$ & $\mathrm{a}^{\mathrm{th}}$ & $\mathrm{a}^{\exp }$ \\
\hline $2 \mathrm{~s}_{1 / 2}$ & -0.198143 & -0.198142 & 402.47 & 401.75 \\
$2 \mathrm{p}_{1 / 2}$ & -0.130226 & -0.130236 & 45.96 & 46.17 \\
$2 \mathrm{p}_{3 / 2}$ & -0.130225 & -0.130235 & -3.03 & -3.07 \\
\hline \hline
\end{tabular}

The energies are now seen to agree with the spectroscopic measurements to five figures, while the hyperfine constants agree with measurement at the $0.1 \%$ level. Interestingly, the transition amplitudes between $2 s$ and $2 p$ states, calculated with the all-orders state vectors, disagree with benchmark experiments at the level of $0.3 \%$, bringing into question the accuracy of the experiments!

Work along these lines is continuing, with the goal of predicting properties of cesium at a level of $0.5 \%$ or better.

\section{REFERENCES}

/1/ Sucher, J., Phys. Rev. A22 (1980) 348.

/2/ Mittleman, M., Phys. Rev. A24 (1981) 1167.

/3/ Blundell, S.A., Guo, D.S., Johnson, W.R. and Sapirstein, J., At. Data Nucl. Data Tables 37 (1987) 103 .

/4/ Johnson, W.R., Blundel1, S.A. and Sapirstein, J. Phys. Rev. A37 (1988) 307 .

/5/ Johnson, W.R., Idrees, M. and Sapirstein, J., Phys. Rev. A35 (1987) 3218.

16/ Johnson, W.R., Blunde11, S.A. and Sapirstein, J., Phys. Rev. A37 (1988) 2764.

/7/ Blundel1, S.A., Johnson, W.R. and Sapirstein, J., Phys. Rev. A38 (1988) 4961.

18/ Blundell, S.A., Johnson, W.R., Liu, Z.W. and Sapirstein, J., Phys. Rev. A39 (1989) 3768 .

19/ Blundell, S.A., Johnson, W.R., Liu, Z.W. and Sapirstein, J., Phys. Rev. A40 (1989) 2233. 